\title{
In Memory of Professor Peter Dmitrievich Krutko (1932-2010)
}

\author{
DOI: $10.3103 / \mathrm{S} 1052618811010092$
}

Peter Dmitrievich Krutko was born on June 27, 1932, on a farm in the Stalingrad steppe. In 1946, he graduated from a seven-year school and entered the Stalingrad Air Force training school. In 1949, as a medalist graduate of the special school, he was sent to the Zhukovskii Air Force Engineering Academy. In 1955, he graduated from the Faculty of Radio Engineering, and then completed one-year courses in computer engineering.

Krutko's scientific work began in 1956 at the Department of Airborne Weapons in the newly established Air Force Research Institute, whose purpose was to use computer technology to solve military aviation problems. Krutko's duties and research interests were associated with research on the application of computer technologies in onboard complexes of aircrafts that had different purposes. He developed hardware architecture for the automatic input of radar data into digital computers, suggested methods for the digital processing of these data, tracking of air targets, and their selection through support channels (the results of these studies found practical application in the development of samples of onboard aiming and firing systems, as well as in the cre-

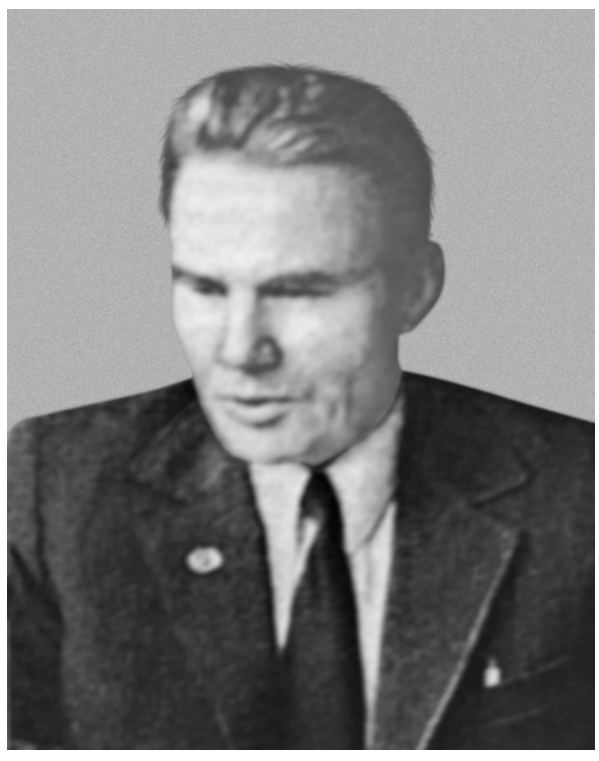
ation of a radar station for air surveillance and air traffic control).

In the early 1960s, Krutko developed statistical methods for studying the dynamics and assessing the accuracy of nonstationary and highly nonlinear systems whose structures contain discrete elements and digital calculators. At the same time, research on the development of methods of synthesis of digital control systems was being carried out. To solve these problems, Krutko developed a mathematical apparatus that was a discrete analogue of classical variational calculus. His theoretical results were published in two monographs, Statistical Dynamics of Impulsive Systems (1963) and Variational Methods for the Synthesis of Digital Controllers (1968). During this period Krutko carried out applied research on the justification and development of control systems for various purposes. In particular, the application of methods of the developed theory of digital systems provided a solution to control problems of front-line aviation weapons for the destruction of ground-based radar units and antiaircraft guided missiles, as well as the development and creation of the first digital autopilots in collaboration with industrial organizations.

Under the leadership of Krutko, applied research was performed in which important scientific and technical results were obtained on the development of algorithmic support of basic onboard complexes of flight navigation and control of different types of aircraft and spacecraft. For the first time in the country, work on aerospace aircraft navigation was carried out based on the use of artificial satellites.

Krutko completed his postgraduate course ahead of schedule, in two years, completing his candidate's dissertation in 1962. He defended his doctoral thesis in 1966.

While engaged in educational work, Krutko continued active research in the field of automation of control processes. He established properties of symmetry in automatic control systems and grounded ideas, development of which was the basis of a new scientific direction in the theory of dynamic systems.

As a result of theoretical research methods developed for analytical design of linear and nonlinear tracking systems of high dynamic accuracy, problems of algorithm synthesis with almost optimal performance were solved; problems of damping of self-oscillations in systems were addressed, the structure of which contains highly nonlinear elements; methods were developed for the synthesis of algorithm structures of the coordinated and autonomous in the degrees of freedom motion control of multidimensional systems; and procedures were developed of the synthesis of control algorithms for complex systems based on the model of nonlinear dynamics without classical linearizing of the original equations. 
In the applied sense, methods for the inverse dynamics problems are the theoretical and methodological basis for the effective design technology of algorithmic support of management systems for various purposes. Using these methods, problems of flight control of aircraft of different classes, and movement and manipulation of mobile robots, underwater vehicles, and other technical systems were solved.

As a result of theoretical and applied research, Krutko published over 120 articles in journals of the Academy of Sciences, three monographs with the publishing house Nauka (1987, 1988, and 1991), and one with the publishing house Radio and Communication (1987) in collaboration with A.I. Maximov and L.M. Skvortsov.

Krutko constantly combined research activities with scientific and organizational work. From 1967, he was a member of the editorial board and then for many years a deputy editor of the journal Proceedings of the Academy of Sciences of the Soviet Union. Technical Cybernetics. From 1968, Krutko was a member of the Scientific Council of the Soviet Union on the problems of traffic management, and in 1984 he became a member of the National Committee of the Soviet Union on Automatic Control and a Vice Chairman of the Scientific Committee on Theory. From 1996, and until his last days, Krutko was a member of the editorial board of the journal Problems of Mechanical Engineering and Machine Reliability.

The most important area of his activities was the training of scientific personnel. Among his student are 9 doctors and 28 candidates of science.

Bright memory of Krutko will remain forever in the hearts of those who knew and loved him. 